\title{
The phenotype of recurrent 10q22q23 deletions and duplications
}

\author{
Bregje WM van Bon ${ }^{\star 1,22}$, Jorune Balciuniene ${ }^{2,22}$, Gary Fruhman ${ }^{3,22}$, Sandesh Chakravarthy Sreenath Nagamani ${ }^{3}$, \\ Diane L Broome ${ }^{4}$, Elizabeth Cameron ${ }^{5}$, Danielle Martinet ${ }^{6}$, Eliane Roulet ${ }^{7}$, Sebastien Jacquemont ${ }^{6}$, \\ Jacques S Beckmann ${ }^{6,8}$, Mira Irons ${ }^{9}$, Lorraine Potocki ${ }^{3}$, Brendan Lee ${ }^{3,10,11}$, Sau Wai Cheung ${ }^{3}$, Ankita Patel ${ }^{3}$, \\ Melissa Bellini ${ }^{12}$, Angelo Selicorni ${ }^{13}$, Roberto Ciccone ${ }^{14}$, Margherita Silengo ${ }^{15}$, Annalisa Vetro ${ }^{14}$, Nine V Knoers ${ }^{1}$, \\ Nicole de Leeuw ${ }^{1}$, Rolph Pfundt ${ }^{1}$, Barry Wolf ${ }^{5,16}$, Petr Jira ${ }^{17}$, Swaroop Aradhya ${ }^{18}$, Pawel Stankiewicz ${ }^{3}$, \\ Han G Brunner ${ }^{1}$, Orsetta Zuffardi ${ }^{15,19}$, Scott B Selleck ${ }^{20,21}$, James R Lupski ${ }^{3,10,11}$ and Bert BA de Vries ${ }^{1}$
}

The genomic architecture of the 10q22q23 region is characterised by two low-copy repeats (LCRs3 and 4), and deletions in this region appear to be rare. We report the clinical and molecular characterisation of eight novel deletions and six duplications within the 10q22.3q23.3 region. Five deletions and three duplications occur between LCRs3 and 4, whereas three deletions and three duplications have unique breakpoints. Most of the individuals with the LCR3-4 deletion had developmental delay, mainly affecting speech. In addition, macrocephaly, mild facial dysmorphisms, cerebellar anomalies, cardiac defects and congenital breast aplasia were observed. For congenital breast aplasia, the NRG3 gene, known to be involved in early mammary gland development in mice, is a putative candidate gene. For cardiac defects, BMPR1A and GRID1 are putative candidate genes because of their association with cardiac structure and function. Duplications between LCRs 3 and 4 are associated with variable phenotypic penetrance. Probands had speech and/or motor delays and dysmorphisms including a broad forehead, deep-set eyes, upslanting palpebral fissures, a smooth philtrum and a thin upper lip. In conclusion, duplications between LCRs 3 and 4 on 10q22.3q23.2 may lead to a distinct facial appearance and delays in speech and motor development. However, the phenotypic spectrum is broad, and duplications have also been found in healthy family members of a proband. Reciprocal deletions lead to speech and language delay, mild facial dysmorphisms and, in some individuals, to cerebellar, breast developmental and cardiac defects.

European Journal of Human Genetics (2011) 19, 400-408; doi:10.1038/ejhg.2010.211; published online 19 January 2011

Keywords: 10q22.3q23.2; NRG3; BMPR1A; PTEN; GRID1; breast development

\section{INTRODUCTION}

Patients with submicroscopic aberration syndromes have traditionally been recognised by a specific combination of clinical features. ${ }^{1}$ When chromosomal banding techniques became available in the 1970s, the cytogenetic basis of many of these syndromes was revealed. ${ }^{2-5}$ The development of subtelomeric fluorescent in situ hybridisation (FISH), targeting all telomeres in a single assay, led to a shift from the original 'phenotype-first' approach to a 'genotypefirst' approach. In the absence of a recognisable phenotype, individuals were screened by FISH for novel submicroscopic chromosomal abnormalities. On the basis of similar genomic aberrations in various patients the clinical presentation was delineated, by a process designated 'reverse phenotypics. ${ }^{6}$

Using new molecular karyotyping techniques such as subtelomeric MLPA and microarray analysis, reverse phenotypics has proven to be successful, by the constantly increasing list of microdeletion/microduplication syndromes. ${ }^{7}$ Some of these have a recognisable phenotype, such as the 17q21.31 microdeletion syndrome. ${ }^{8-10}$ Others, such as deletions of chromosome band 1q21.1,15q13.3 or 16p13.11, give rise to less consistent phenotypes. ${ }^{11-21}$ These aberrations show incomplete penetrance, as demonstrated by their presence in clinically unaffected relatives. Several of these aberrations are associated with an increased

${ }^{1}$ Department of Human Genetics, Radboud University Nijmegen Medical Centre, Nijmegen, The Netherlands; ${ }^{2}$ Department of Biology, Temple University, Philadelphia, PA, USA; ${ }^{3}$ Department of Molecular and Human Genetics, Baylor College of Medicine, Houston, TX, USA; ${ }^{4}$ Department of Medical Genetics SCPMG, Anaheim, CA, USA; ${ }^{5}$ Department of Medical Genetics, Henry Ford Health System, Detroit, MI, USA; ${ }^{6}$ Service of Medical Genetics, Centre Hospitalier Universitaire Vaudois, Lausanne, Switzerland; ${ }^{7}$ Service of Pediatrics, Centre Hospitalier Universitaire Vaudois, Lausanne, Switzerland; ${ }^{8}$ Department of Medical Genetics, University of Lausanne, Lausanne, Switzerland; ${ }^{9}$ Division of Genetics, Department of Pediatrics, Children's Hospital Boston, Harvard Medical School, Boston, MA, USA; ${ }^{10}$ Department of Pediatrics, Baylor College of Medicine, Houston, TX, USA; ${ }^{11}$ Texas Children's Hospital, Houston, TX, USA; ${ }^{12}$ Department of medical genetics, San Carlo Hospital, Milano, Italy; ${ }^{13}$ Clinical Genetics Azienda Ospedaliera San Gerardo, Monza Italy; ${ }^{14}$ Department of Medical Genetics, University of Pavia, Pavia, Italy; ${ }^{15}$ Department of Pediatrics, University of Torino, Torino, Italy; ${ }^{16} \mathrm{Center}$ for Molecular Medicine and Genetics, Wayne State University School of Medicine, Detroit, MI, USA; ${ }^{17}$ Department of Pediatrics, Jeroen Bosch Hospital, 's-Hertogenbosch, The Netherlands; ${ }^{18}$ Clinical Microarray Services, GeneDx, Gaithersburg, MD, USA; ${ }^{19}$ IRCCS C Mondino, Pavia, Italy; ${ }^{20}$ Departments of Pediatrics, and Genetics, Cell Biology and Development, University of Minnesota, Minneapolis, MN, USA; ${ }^{21}$ Department of Biochemistry and Molecular Biology, The Pennsylvania State University, University Park, PA, USA

*Correspondence: Dr BWM van Bon, Department of Human Genetics, 849, Radboud University Nijmegen Medical Centre, PO Box 9101 , Nijmegen 6500 HB, The Netherlands. Tel: +31 24361 3946; Fax: +31 24366 8774; E-mail: B.vanBon@antrg.umcn.nl

22These authors contributed to the paper equally.

Received 15 June 2010; revised 21 September 2010; accepted 21 October 2010; published online 19 January 2011 
risk of neuropsychiatric disorders, such as schizophrenia and autism. ${ }^{15,16,22}$ Chromosomal anomalies, with an unpredictable and inconsistent phenotypic outcome, represent a difficult counselling situation for clinicians. Microarray analysis during pregnancy is now offered in several countries, and prenatal detection of these submicroscopic changes is a particularly challenging counselling situation. ${ }^{23}$ Therefore, the need for extensive information about phenotypic outcomes of such recurrent aberrations is essential.

Recurrent deletions of 10q22.3q23.2 have been characterised by cognitive and behavioural abnormalities. ${ }^{24}$ The $10 \mathrm{q} 22.3 \mathrm{q} 23.2$ region is characterised by a complex set of low-copy repeats (LCRs), which can give rise to various genomic changes mediated by non-allelic homologous recombination (NAHR) ${ }^{25}$ Balciuniene et al ${ }^{24}$ described three probands with interstitial deletions in this region. The breakpoints in two of these mapped within these LCRs (LCR3 and LCR4) and inheritance was reported in one. This deletion segregated with a wide range of cognitive and behavioural phenotypes within a large family. The third proband had a complex non-contiguous rearrangement consisting of two deletions with breakpoints in a unique genomic sequence, with the most proximal breakpoint between LCR3 and LCR4 and a telomeric breakpoint $\sim 630 \mathrm{~kb}$ distal of LCR4. A considerable overlap of cognitive and behavioural phenotypes was noted among probands and affected family members. The presence of LCRs in this region suggests that this locus has an increased susceptibility to chromosomal rearrangements. ${ }^{24}$ In contrast to expectations based on the chromosomal architecture defined by LCR3 and LCR4, only six novel deletions and no duplications comprising this region have been reported. ${ }^{26}$ Of these, four were clinically assessed.

In this report, we describe the clinical and molecular characterisation of eight novel deletions and six duplications within the $10 \mathrm{q} 22.3 \mathrm{q} 23.3$ region. Five deletions and three duplications occur between LCRs 3 and 4, whereas three deletions and three duplications have unique breakpoints.

\section{METHODS}

\section{Patients and DNA samples}

In this study, 14 individuals with an aberration in 10q22q23 were studied. DNA was isolated according to standard procedures. Except for individual 4, who had a 47,XYY karyotype, all others had normal karyotypes by G-banded chromosome studies. Clinical information was obtained from the respective physicians.

The submicroscopic aberrations were detected by array analysis using different array platforms.

All deletions were fine-mapped using high-resolution, custom-tiled oligonucleotide CGH arrays. These were designed, manufactured, processed and analysed by Roche NimbleGen Systems Inc. (Madison, WI, USA). Except for patient 3, all patients were analysed using arrays (design ID 2794) that contained 385000 isothermal oligonucleotide probes finely tiled across the human chromosome 10 region from 44 to $92 \mathrm{Mb}$ (human genome reference sequence version hg18). Data analysis included 371000 'unique' probes providing an average coverage of one probe every 130 nucleotides. A probe was considered 'unique' if it differed by at least five nucleotides from homologous hits retrieved by a Blastn search against the entire human genome. Less stringent criteria for 'uniqueness' were applied for probes in LCR sequences, and probes showing only one nucleotide mismatch to paralogous sequences were included for the analysis. Patient 3 was analysed using a different design array (design ID 5621). The chromosome 10 region from 77 to $92 \mathrm{Mb}$ (human genome reference sequence version hg18) was finely tiled with 68291 probes, which yielded a mean coverage of one probe per 210 nucleotides. All probes on this array were 'unique', on the basis of the aforementioned criteria. Each sample was subjected to one hybridisation experiment, except for patient 2 whose DNA was subjected to two independent hybridisation arrays. Test DNA samples were labelled with Cy3 and paired with a reference DNA sample labelled with Cy5. We used a human DNA reference sample (Promega) provided by Roche NimbleGen Systems, which is a pool of genomic DNA from six anonymous males. Probe hybridisation signals were expressed as the $\log 2$ ratio of signal intensities of a test sample versus signal intensities of a reference sample. Array data were analysed with the segMNT or DNACopy algorithms. More detailed description of probe design, array construction, DNA labelling, hybridisation and data analysis can be found on the Roche Nimblegen Systems webpage (http://www.nimblegen.com/products/ lit/index.html).

In the case of copy number variations (CNVs) with breakpoints mapping within LCRs, the automated segmentation algorithm performed poorly. The breakpoints assigned by analyses of different averaging windows were inconsistent and fell within large genomic intervals up to $5 \mathrm{Mb}$ in length. Instead, we visualised the data using our previously published colour binning method and compared the data of our patients with those from three previously obtained control samples. ${ }^{24}$ For patient $2, \log 2$ ratios from two independent experiments were averaged, and the averaged values were used for colour binning analysis. Breakpoint genomic coordinates provided in the result section represent our best estimate, and are in reference to the hg18 version of the Human Genome assembly (http://genome.ucsc.edu/).

\section{RESULTS}

\section{Molecular findings}

The breakpoints of the deletions and duplications are shown in Figures 1 and 2, respectively. In addition to the aberration in the $10 \mathrm{q} 22 \mathrm{q} 23$ region, patient 3 had a de novo $722 \mathrm{~kb}$ gain in $2 \mathrm{q} 36.3 \mathrm{q} 36.3$ (167.2-167.7 Mb), patient 4 a 47,XYY karyotype, patient 12 a de novo $1.5 \mathrm{Mb}$ deletion at $16 \mathrm{p} 13.11(14.8-16.3 \mathrm{Mb})$ and patient 14 an inherited 2.2 Mb gain in 6q25.1 (149.4-151.6 Mb). All 10q22q23 and additional aberrations are summarised in supplementary Table A.

\section{Clinical data}

The clinical findings of the patients are described in detail in Supplementary information. The clinical features of probands 1-5 with an LCR3-4 deletion are summarised in Table 1 and of probands 10-12 with an LCR3-4 duplication in Table 2. The latter table also includes clinical features of patient 9 , who had a largely overlapping duplication with breakpoints located in unique sequences outside LCRs 3 and 4 . The clinical features of probands $6-8$ and of probands 13-14 are displayed in Tables 3 and 4, respectively. Photographs of six patients (cases 1, 3, 4, 9, 10 and 11), whose parents consented for publication, are shown in Figure 3.

\section{DISCUSSION}

In recent years, many recurrent rearrangements have been added to the increasing list of genomic disorders, ${ }^{27}$ including deletions in the 10q22.3q23.2 region. ${ }^{24}$ The breakpoints of this deletion are flanked by LCRs. LCR3 flanks the proximal breakpoint and harbours two large ( $>300 \mathrm{~kb}$ ) highly homologous ( $99.8 \%$ identity) segmental duplications. They are composed of smaller modules with different orientations that are dispersed elsewhere on chromosome 10 and on other chromosomes. LCR4 flanks the distal breakpoint and contains $\sim 170 \mathrm{~kb}$ of sequence homologous to LCR3, as well as $>100 \mathrm{~kb}$ of sequence homologous to LCRs located near the chromosome 10 centromere. ${ }^{24}$ On the basis of this genomic architecture, an increased susceptibility of chromosomal rearrangements between LCRs 3 and 4 can be expected in this region. However, since the description of two deletions by Balciuniene et al, ${ }^{24}$ only six additional cases have been reported. ${ }^{26}$ The additional five new cases with the LCR3-4 deletion in the current study were detected within a cohort of 30991 individuals with MR and/or multiple congenital anomalies, leading to a frequency 
Chromosome 10

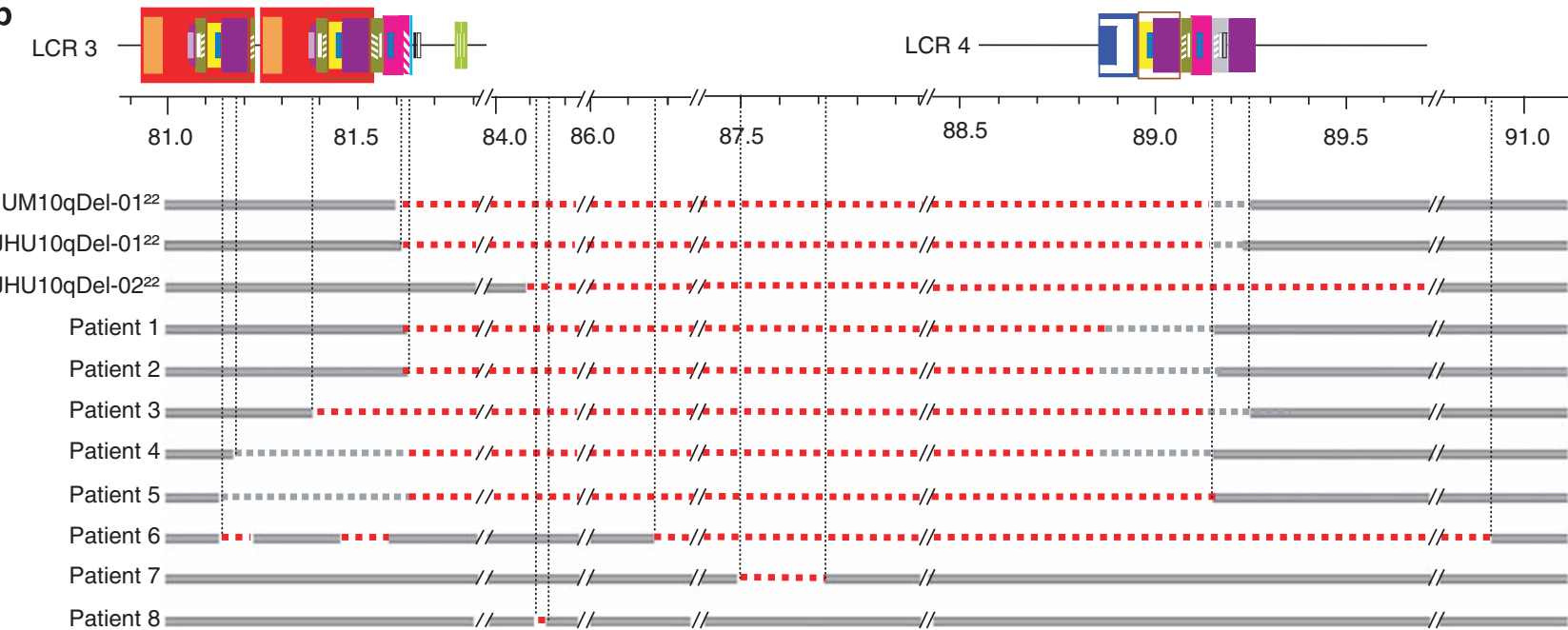

Genes

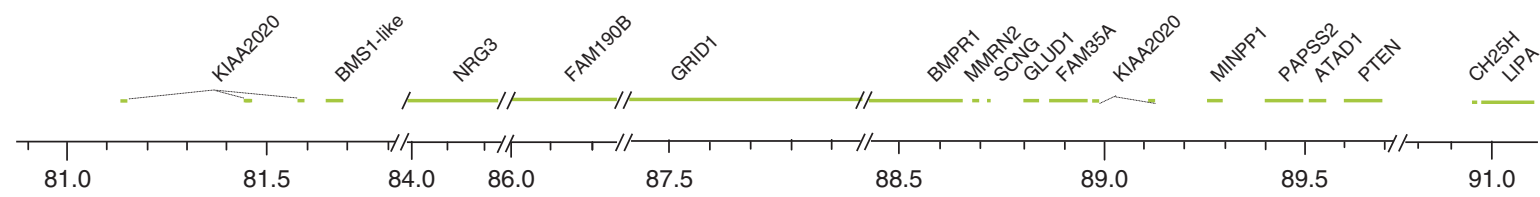

Figure 1 Schematic representation of $10 \mathrm{q}$ genomic rearrangements identified in the probands. (a) Structure of LCRs located in 10q22.3-q23.2 is shown. ${ }^{24}$ Blocks of the same colour and/or pattern denote paralogous sequences. Degree of sequence identity between paralogous sequences ranges from 90.8 to 99.8\%. Genomic position (in Mb) is shown on a scale below the LCRs, and corresponds to human reference human genome reference sequence version hg18. (b) Map of deletions predicted by oligonucleotide CGH arrays in patients 1-8 and of previously studied individuals JHU10qDel-01, JHU10qDel-02, UM10qDel-01. ${ }^{24}$ Grey bar indicates intact DNA sequence. Red dashed line indicates hemizygous deletion. Grey dashed line denotes the genomic area that contains a breakpoint. Genes located in this genomic region are shown below the diagram of the rearrangements.

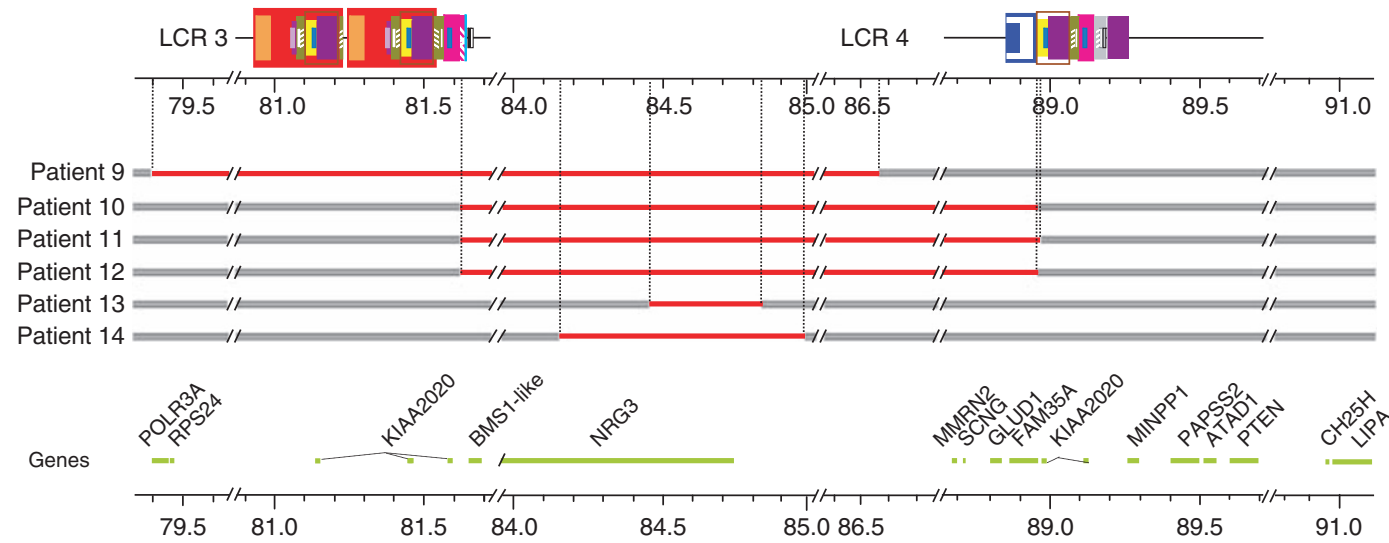

Figure 2 Map of duplications predicted by oligonucleotide CGH arrays in patients 9-14. Grey bar indicates intact DNA sequence. Red solid line indicates duplication. Genes located in this genomic region are shown below the diagram of the rearrangements. Genomic position (in Mb) is shown on a scale below the LCRs, and corresponds to human reference human genome reference sequence version hg18.

of $0.016 \%$. This is a much lower frequency than reported for other genomic disorders, such as Williams syndrome $(0.31 \%),{ }^{28}$ proximal $16 \mathrm{p} 11.2$ deletions $(0.6 \%),{ }^{29} 17 \mathrm{q} 21.31$ deletions $(0.64 \%)^{9}$ and $15 \mathrm{q} 13.3$ deletions $(0.24 \%){ }^{21}$

On the basis of genomic architecture, the lower frequency might be explained by the $\sim 7 \mathrm{Mb}$ unique sequence between the LCRs in the
$10 \mathrm{q} 22.3 \mathrm{q} 23.2$ region, which is much larger than the distance between segmental duplications in other LCR-mediated recurrent rearrangements reported thus far. The distance between two LCRs is known to be one of the genomic architectural features that influences the efficiency of NAHR. ${ }^{30}$ In fact, on the basis of the 18 new genomic disorders described since 2005, recently reviewed by Mefford and 


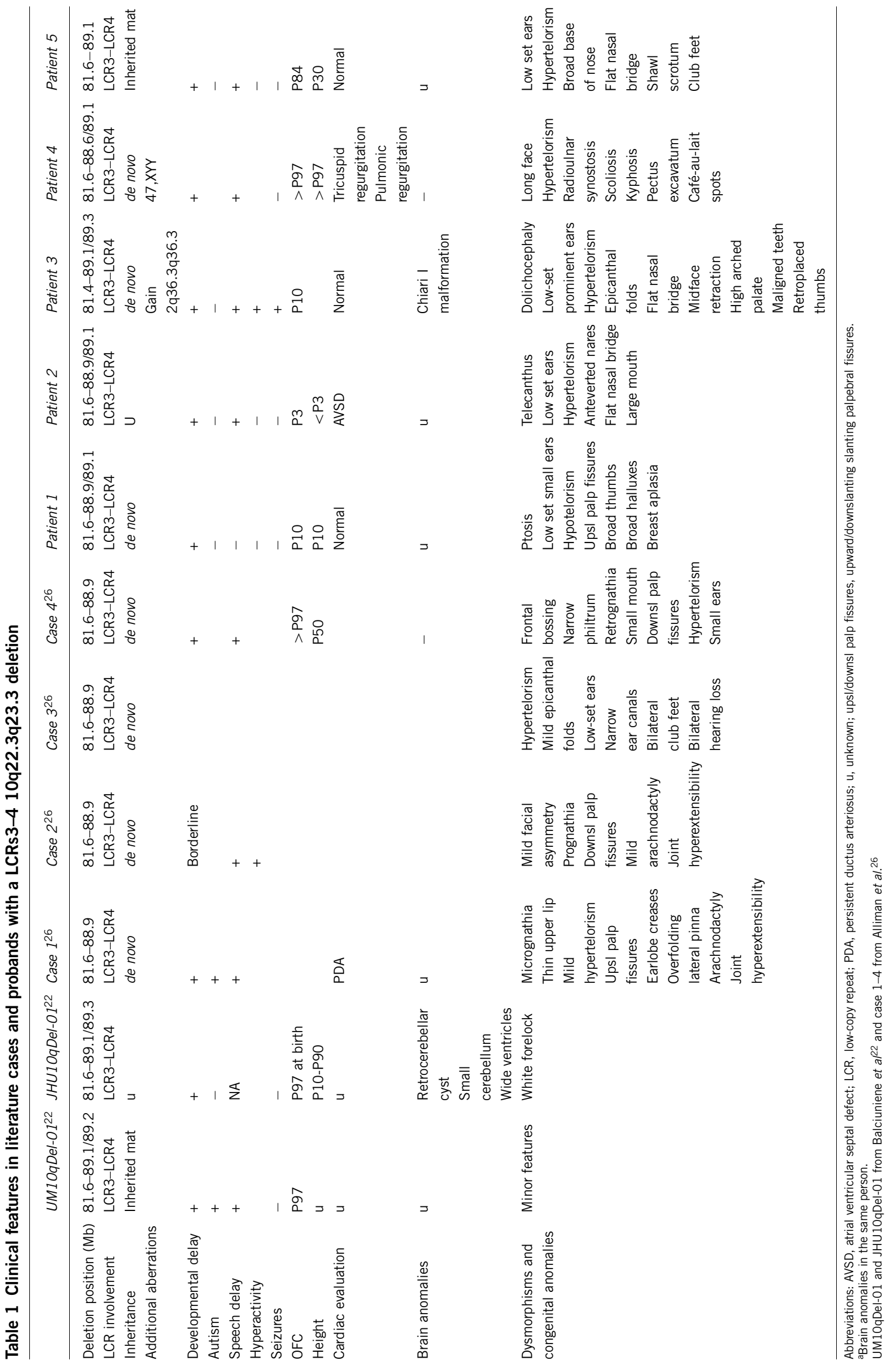


Table 2 Clinical features of four patients with a duplication of $10 \mathrm{q} 22.3 \mathrm{q} 23.2$

\begin{tabular}{|c|c|c|c|}
\hline & \multicolumn{3}{|c|}{ Sibpair patients } \\
\hline & Patient 9 & $10-11$ & Patient 12 \\
\hline Duplication position (Mb) & $79.4-86.6$ & $81.6-89.0$ & $81.6-89.0$ \\
\hline LCR involvement & No & LCR3-CR4 & LCR3-LCR4 \\
\hline Inheritance & Unknown & Inherited mat & de novo \\
\hline Additional aberrations & - & - & $16 p 13.1 \mathrm{del}$ \\
\hline Developmental delay & + & + & + \\
\hline Speech delay & + & + & - \\
\hline Seizures & - & & - \\
\hline Birth weight & P50 & & P20 \\
\hline Recurrent ear infections infancy & + & & \\
\hline Current weight & P30 & & P25 \\
\hline Current height & P50 & & $>$ P90 \\
\hline Current OFC & P50 & & P25 \\
\hline Behavioural problems & $\begin{array}{l}\text { Impaired social } \\
\text { interaction }\end{array}$ & & $\begin{array}{l}\text { Impaired social } \\
\text { interaction }\end{array}$ \\
\hline Deep-set eyes & + & + & + \\
\hline Upslanting palpebral fissures & + & + & - \\
\hline Hypotelorism & - & + & + \\
\hline Low and prominent ears & + & - & - \\
\hline Smooth philtrum & + & + & - \\
\hline Thin upper lip & + & + & + \\
\hline Full lower lip & + & - & + \\
\hline Strabismus & - & + & - \\
\hline Micrognathia & - & + & - \\
\hline Full cheeks & + & - & + \\
\hline Large wide spaced teeth & + & + & \\
\hline Thin built & - & + & - \\
\hline
\end{tabular}

Abbreviation: LCR, low-copy repeat.

Three patients with an LCRs3-4 duplication and one patient with a duplication in unique sequences, but sharing $5 \mathrm{Mb}$ of overlap with other three duplications.

Table 3 Clinical features in probands with a 10q22.3q23.3 deletion, with breakpoints in unique sequences

\begin{tabular}{llll}
\hline & Patient 6 & Patient 7 & Patient 8 \\
\hline Deletion position (Mb) & $86.2-90.9$ & $87.5-87.7$ & $84118-84148$ \\
LCR involvement & None & None & None \\
Inheritance & de novo & $\mathrm{u}$ & Inherited pat \\
Additional aberrations & - & - & - \\
Developmental delay & - & - & + \\
Autism & - & - & + \\
Hyperactivity & + & - & - \\
Seizures & - & - & + \\
OFC & $>$ P97 & P95 & P10 \\
Height & P70 & <P3 & P50 \\
Cardiac evaluation & u & AVSD & u \\
Brain anomalies & u & NA & $\mathrm{u}$ \\
Dysmorphisms and & Upturned nose & Prominent forehead & - \\
congenital anomalies & Gl polyps & Downsl palp fissures & \\
& Hypertelorism & Hypertelorism & \\
& & Anteverted nares & \\
& & Small low set ears & \\
& & Diaphragmatic & \\
& & eventration & \\
& & Undescended testes &
\end{tabular}

Abbreviations: downsl palp fissures, downslanting palpebral fissures; GI, gastrointestinal; LCR, low-copy repeat; u, unknown.
Table 4 Clinical features of probands with small sized 10q22.3q23.3 duplications with breakpoints in unique sequences

\begin{tabular}{lcc}
\hline & Patient 13 & Patient 14 \\
\hline Duplication position $(\mathrm{Mb})$ & $84.5-84.8$ & $84.2-85$ \\
LCR involvement & No & No \\
Inheritance & Inherited pat & Inherited pat \\
Additional aberrations & - & Inh 6q25.1 dup \\
Developmental delay & - & - \\
Speech delay & (Blindness related) & - \\
Seizures & - & - \\
Birth weight & P50 & $<$ P3 \\
Current height & P10 & P75 \\
Current weight & P3 & $>$ P97 \\
Deep-set eyes & + & - \\
Full lower lip & + & - \\
Strabismus & + & - \\
Anteverted nares & + & - \\
Full cheeks & + & - \\
Urogenital anomalies & - & + \\
Cardiac defect & - & + \\
Fusion of sacral vertebrae & - & + \\
Long slender ribs and clavicles & - & + \\
\hline Abbreiation: LCR low-cop & & +
\end{tabular}

Abbreviation: LCR, low-copy repeat.

On the basis of phenotype and inheritance pattern, these aberrations most likely represent non-pathogenic variants.

Eichler, ${ }^{31}$ the distance between LCRs3 and 4 on $10 \mathrm{q} 22.3 \mathrm{q} 23.2$ is the largest in size (Figure 4). Except for the 16p11.2p12.2 deletion, having a size of $7 \mathrm{Mb}$, all aberrations are $<2.5 \mathrm{Mb}$. However, the latter study did not include previously described LCR-mediated syndromes delineated through a 'phenotype-first approach'. Some of these also include intermediate-sized deletions, such as the Williams (MIM no. 19450), velocardiofacial (MIM no. 192430), SmithMagenis (MIM no. 182290), Prader-Willi (MIM no. 176270) and Angelman (MIM no. 105830) syndromes (Figure 4). ${ }^{31-36}$ In addition, it has been observed that larger-sized genomic rearrangements, using LCRs positioned farther apart, are regularly associated with larger LCRs. ${ }^{30}$ Although the aberration on $10 \mathrm{q} 22.3 \mathrm{q} 23.2$ is largest in size, its LCRs are intermediate in size compared with LCRs in other genomic rearrangements.

The low frequency of 10q22.3q23.2 deletions among MR/MCA individuals may reflect lack of ascertainment because of the mild phenotype in some individuals with this deletion. Balciuniene et a ${ }^{24}$ reported multiple family members having this deletion, which segregated with a wide range of cognitive and behavioural phenotypes, including learning difficulties, speech and language delay, ADHD and autism. Individuals with a similar mild phenotype will often not be tested for submicroscopic aberrations. Similar phenotypic variability has been described for other rearrangements, such as on chromosomes 1q21.1, 15q13.3 and 16p13.11. All aberrations are enriched in affected persons compared with control individuals. ${ }^{12-16,18-21}$ A less likely explanation for the low frequency might be that 10q22.3q23.2 deletions in foetuses often lead to miscarriages. Although miscarriages were not reported in our families, the family reported by Balciuniene et al ${ }^{24}$ showed spontaneous abortions and a stillbirth.

In addition to the six clinically reported $10 \mathrm{q} 22.3 \mathrm{q} 23.2$ cases, we present the clinical features of five novel patients with this deletion. Of these 11 deletions, seven had arisen de novo, two were inherited and for two inheritance remained unknown. Two patients had additional 
A
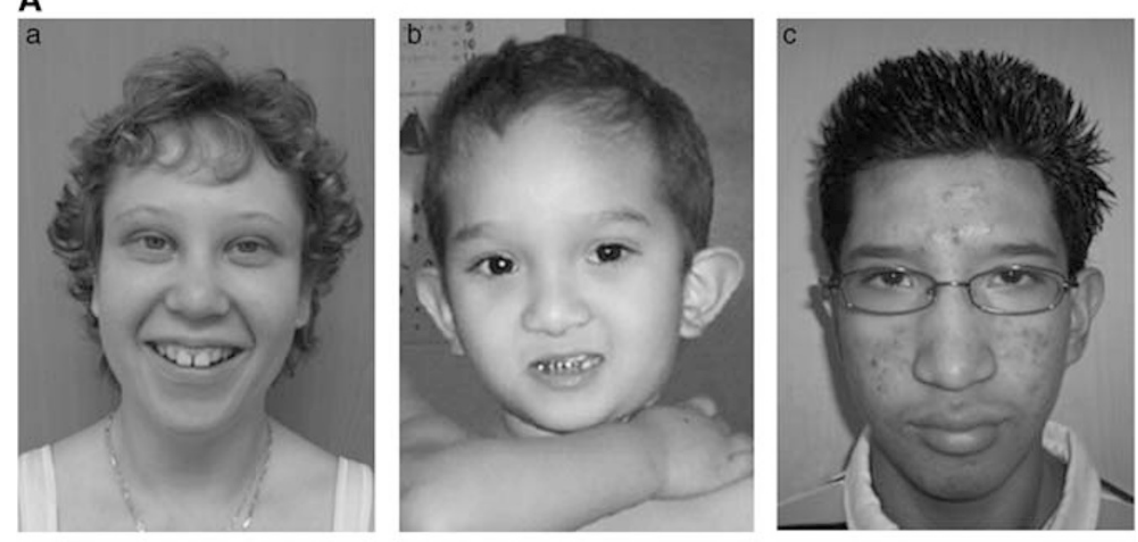

B
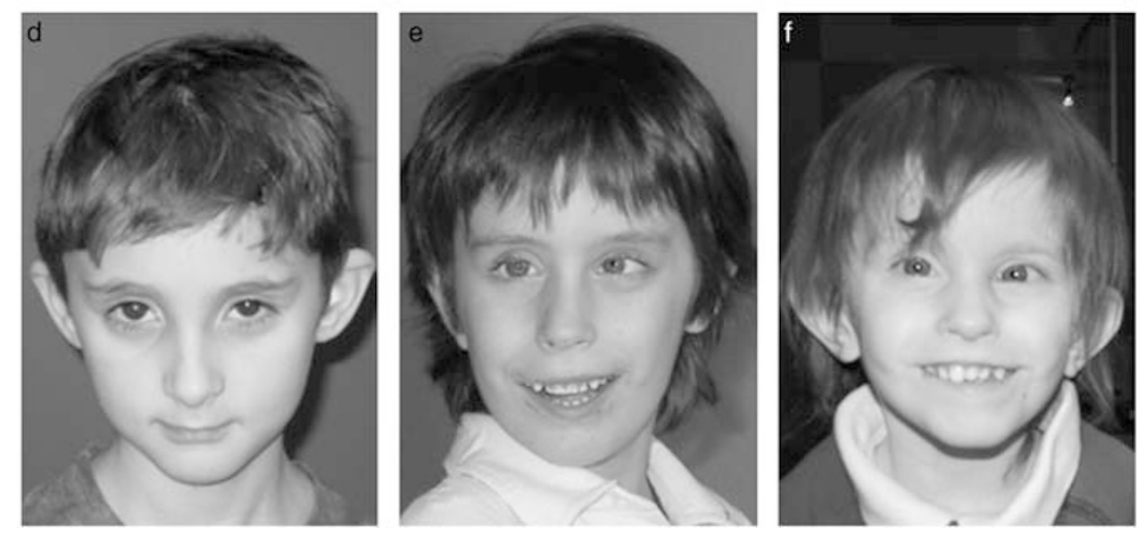

Figure 3 (A) Photographs of probands 1, 3 and 4 with deletions between LCR3 and LCR4. Proband 1 (a) at 13 years of age; she has upward-slanting palpebral fissures, hypotelorism and low-set ears. Proband 3 (b) at 3 years and 7 months of age; he has dolichocephaly, low-set and prominent ears, hypertelorism, epicanthal folds and a flat midface. Proband 4 (c) at 12 years of age; he has hypertelorism, almond-shaped eyes, low-set ears and full lips. (B) Photographs of patients 9, 10 and 11 with duplications of 10q22.3q23.2. Patient 9 (d) at 9 years of age; he has a triangular face, a broad forehead, upslanting palpebral fissures, slightly deep-set eyes, lateral flaring of eyebrows, prominent ears with thickened horizontal superior helices, a thin upper lip and a smooth philtrum. Sibling patients 10 (e) and 11 (f) show upslanting palpebral fissures, strabismus, hypotelorism, a smooth philtrum and anteverted nares.

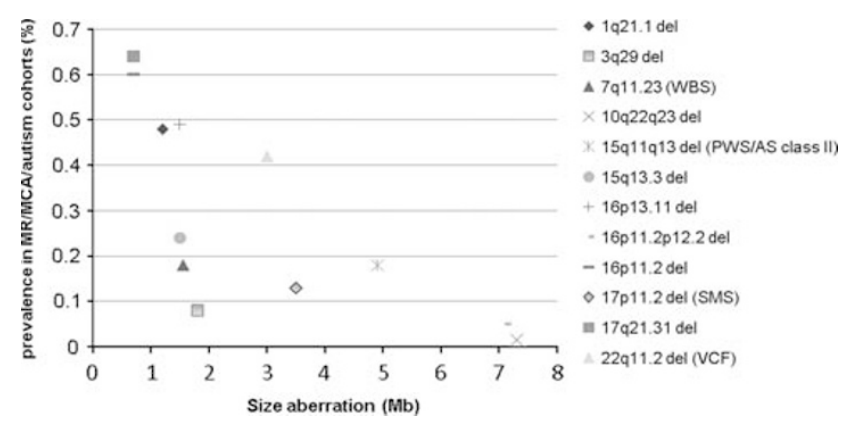

Figure 4 Frequency of LCR-mediated microdeletions in cohorts of individuals with MR, multiple congenital anomalies and/or autism ( $y$ axis) $9,12,20,21,29,32,74,75$ compared with the size of the unique sequence between LCRs ( $x$ axis). ${ }^{31-36}$ WBS, Williams-Beuren Syndrome; PWS, Prader Willi Syndrome; AS, Angelman syndrome; SMS, Smith-Magenis syndrome; VCF, velocardiofacial syndrome. Frequencies of PW/AS and VCF syndromes were corrected for the most common deletion causing these syndromes.

chromosome abnormalities. The phenotype of patient 2 might have been influenced by the additional de novo duplication of chromosome $2 \mathrm{q} 36.3$. However, this aberration has also been reported in a healthy father of an affected individual (DECIPHER database ID 248304), and several partially overlapping duplications in this region have been reported in the Database of Genomic Variants. The phenotype of patient 4 might have been influenced by the 47,XYY karyotype. In general, individuals with an XYY karyotype have lower IQ scores, mainly verbally, as expected for their social background, but not impaired in relation to general population norms. In addition, problems with anger control and also with attention have been described. ${ }^{37}$

Cognitive development was impaired in all 11 probands, varying from mild to moderate impairment. All patients had apparent speech and language problems. Three patients also had motor developmental delay, but their speech was more severely affected than their motor development.

Patients were mildly dysmorphic, and low-set ears, hypertelorism and a flat nasal bridge were frequently noted. Head circumference ranged from the 3 rd centile to above the 97 th centile, but the majority of patients were macrocephalic.

Behavioural problems were present in some, but were non-overlapping in type; two patients had autism, ${ }^{24,26}$ two showed hyperactivity $^{26}$ and patient 4 demonstrated aggressive behaviour. In the latter, this may have been due to his $47, \mathrm{XYY}$ karyotype. 
Two patients had cerebellar anomalies; low-lying cerebellar tonsils in patient 3 and a retrocerebellar cyst with a small cerebellum in patient JHU10qDel-01 were reported by Balciuniene et al. ${ }^{24}$

Patient 1 had unilateral congenital breast aplasia. Except for two patients, all other LCR3-LCR4 deletion probands were male. Patients 2 and 4 and case 1 of Alliman et al had a cardiac defect (AVSD, tricuspid and pulmonic regurgitation and persistent ductus arteriosus, respectively) and patient 3 had epilepsy. An AVSD was also noted in patient 7 and epilepsy in patient 8 . However, these two latter patients had smaller intragenic deletions, located in unique sequences.

The breakpoints of the deletion in proband 6 were also located in unique sequences, including LCR4 and the PTEN gene. This patient had macrocephaly and juvenile colonic polyps, common features of contiguous gene deletions including tumour suppression genes BMPR1A and PTEN. ${ }^{38}$ Germline loss-of-function heterozygous mutations in PTEN have been described, causing an overlapping phenotype including macrocephaly and gastrointestinal polyps in patients with Cowden and Banayan-Riley-Ruvalcaba syndrome. ${ }^{39}$ Furthermore, heterozygous germline loss-of-function mutations in BMPR1A cause juvenile polyposis. ${ }^{40}$

Three currently reported patients $(2,4$ and 7$)$ had a cardiac defect, indicating that a dosage-sensitive gene or genes involved in the pathogenesis of cardiac defects may be located in the 10q22.3q23.2 region. There are two interesting candidate genes located in this region, BMPR1A and GRID1. BMPR1A is deleted in patients 3 and 4 and in all individuals who have been reported with overlapping deletions and cardiac septal defects. ${ }^{41-45}$ Of these deletions, at least one did not include the GRID1 gene. ${ }^{42}$ Cardiac-specific deletion of $B M P R 1 A$ disrupts cardiac morphogenesis in mice, showing ventricular septum, trabeculae, compact myocardium and endocardial cushion defects. $^{46}$

$B M P R 1 A$ was not deleted in patient 7 with a cardiac defect, who had an intragenic deletion of GRID1, which is located only about $500 \mathrm{~kb}$ upstream of BMPR1A. The inheritance of this deletion was unknown, hampering conclusions on pathogenicity. Similar deletions have not been reported in the Database of Genomic Variants, nor in the chromosomal databases DECIPHER and ECARUCA. GRID1 encodes for a subunit of glutamate receptor channels, which have a role in mediating excitatory synaptic transmission in the central nervous system. ${ }^{47}$ The deletion in patient 7 removes exons 5-8 of the GRID1 gene. The removal of these exons would lead to an in-frame protein that would partly lack the extracellular ligand-binding domain, and therefore may represent a dominant-negative mutation. A recent metaanalysis of genome-wide association data, aimed to identify common genetic variants associated with cardiac structure and function, proposed GRID1 as a candidate gene for left ventricle wall thickness. ${ }^{48}$

Remarkably, this glutamatergic gene has also been associated with an entirely different pathology, namely schizophrenia. ${ }^{49-52}$ The glutamatergic system is the major excitatory neurotransmitter system in the central nervous system, ${ }^{53}$ and changes in this system are the basis of the 'glutamate hypothesis' aiming to explain symptoms of schizophrenia. ${ }^{54,55}$ After finding linkage evidence for schizophrenia in 10q22 in different populations, ${ }^{56-59}$ GRID1 was implicated as being the causative gene in several association studies. ${ }^{49-52}$

Another gene in the 10q22 region, associated with schizophrenia risk and presentation, is neuregulin 3 (NRG3). ${ }^{56}$ This gene is located $2.6 \mathrm{Mb}$ proximal of GRID1 and affected in copy number variation in 12 patients in this study. Neuregulins are signalling proteins that mediate cell-cell interactions in the nervous system, heart, breast and other organ systems. ${ }^{60}$ Although several family members of proband 8 had a psychiatric disorder, the intragenic deletion found in the proband and his father did not segregate in the rest of the family. In addition, this intragenic deletion minimally overlaps a CNV (loss) described in two healthy individuals ${ }^{61}$ and in our patient 7 ; however, it extends beyond the CNV telomeric boundary. Therefore, the pathogenic significance of this $\mathrm{CNV}$ remains uncertain.

Taking into account that most probands in this study are still young, definitive evidence linking haploinsufficiency of NRG3 or GRID1 to psychiatric disease could not be established.

$N R G 3$ is also a candidate gene for congenital breast aplasia, which was present in patient 1 . NRG3 has been implicated in early mammary gland development, and $\mathrm{Nrg} 3$ signalling in mice promotes differentiation of mammary epithelial cells at the initial stages of organ formation. Mice harbouring a hypomorphic allele of Nrg3 exhibit defects in mammary gland formation, such as hypoplastic and supernumerary mammary gland formation. ${ }^{62,63}$

$N R G 3$ was also involved in two patients with an intragenic NRG3 duplication and congenital anomalies. Patient 13 had vision problems, most likely because of prematurity, whereas patient 14 had tetralogy of fallot, hypospadias and ambiguous genitalia. Both patients inherited the duplication from an unaffected parent. Although a possible role in organ development cannot be excluded, these duplications did not influence neurocognitive functioning and are most likely non-pathogenic variants.

Three patients (10-12) had a duplication between LCR3 and LCR4. Patient 9 had a duplication that did not involve LCR4, but shared a $5 \mathrm{Mb}$ overlap with the three LCR3-4 duplications. Sibpair patients 10 and 11 inherited this duplication from their healthy mother. The duplication in patient 12 occurred de novo. This patient also had a de novo 16p13.11 deletion, which has been reported as a risk factor, strongly predisposing for, but not sufficient to cause, autism, epilepsy and mental retardation. ${ }^{13,20}$ Duplications involving the 10q22-q23 region are rare. To date, only four cases overlapping this region have been reported. ${ }^{64-67}$ Two of these patients had microcephaly and three had cardiac defects. Precise genotype-phenotype correlations are hampered because these aberrations have often been detected by conventional cytogenetic techniques. In addition, except for one case reported by Dufke et al, ${ }^{65}$ these duplications were much larger in size. Patients 9 and 12 displayed speech delay and impaired social interaction. Sibpair patients 10 and 11 were mentally retarded. Probands 9-11 and the patient described by Dufke et al had a strikingly similar facial appearance including a broad forehead, deep-set eyes, upslanting palpebral fissures, a smooth philtrum and a thin upper lip.

As could be noted in the healthy mother of sibpair patients 10 and 11, duplication of the LCR3-4 region does not always lead to an abnormal phenotype. Incomplete penetrance of chromosomal aberrations is a well-known phenomenon, which has been described for several abnormalities such as 1q21.1, 15q13.3, 16p13.11 and 22q11.2 aberrations, ${ }^{11-14,20,21,68-71}$ with reciprocal duplications of the same interval often less penetrant. ${ }^{72,73}$ To determine the full spectrum and penetrance of the LCR3-4 duplication, more information about probands carrying this duplication, and especially information about other carriers in their families, is needed.

In conclusion, duplications of the LCR3-LCR4 region on $10 \mathrm{q} 22.3 \mathrm{q} 23.2$ are associated with variable phenotypic penetrance. In affected individuals, these aberrations may lead to a distinct facial appearance and developmental speech and/or motor function delays. Deletions between LCRs3 and 4 are clinically penetrant in all cases reported thus far. These deletions are associated with cognitive impairment, mainly affecting speech development, and cardiac defects. In addition, these deletions may also be associated with cerebellar and breast developmental defects. 


\section{CONFLICT OF INTEREST}

The authors declare no conflict of interest.

\section{ACKNOWLEDGEMENTS}

We thank all the parents and children who participated in this study. This work was supported by a grant from the European commission: AnEUploidy project (LSHG-CT-2006-037627) under FP6 (BvB, HB, BBAdV), supplemental grants from The Netherlands Organisation for Health Research and Development (ZonMW 917-86-319 to BBAdV), Hersenstichting Nederland (BBAdV), Cure Autism Now (SBS), the Harrison Endowment and Autism Initiative Funds (JB, SBS).

\section{WEB RESOURCES}

Human Genome Browser: http://genome.ucsc.edu/cgi-bin/hgGateway (for March 2006 assembly)

DatabasE of Chromosomal Imbalance and Phenotype in Humans using Ensembl Resources: https://decipher.sanger.ac.uk

Database of Genomic Variants: http://projects.tcag.ca/variation/?source $=\mathrm{hg} 18$ European Cytogeneticists Association Registered Unbalanced Chromosome Aberrations: www.ecaruca.net

1 Schmickel RD: Contiguous gene syndromes: a component of recognizable syndromes. J Pediatr 1986; 109: 231-241.

2 Ewart AK, Morris CA, Atkinson D et al: Hemizygosity at the elastin locus in a developmental disorder, Williams syndrome. Nat Genet 1993; 5: 11-16.

3 Driscoll DA, Budarf ML, Emanuel BS: A genetic etiology for DiGeorge syndrome: consistent deletions and microdeletions of 22q11. Am J Hum Genet 1992; 50: 924-933.

4 Bowen P, Biederman B, Hoo JJ: The critical segment for the Langer-Giedion syndrome: 8q24.11---q24.12. Ann Genet 1985; 28: 224-227.

5 Ledbetter DH, Riccardi VM, Airhart SD et al: Deletions of chromosome 15 as a cause of the Prader-Willi syndrome. N Eng/ J Med 1981; 304: 325-329.

6 de Vries BB, Winter R, Schinzel A et al: Telomeres: a diagnosis at the end of the chromosomes. J Med Genet 2003; 40: 385-398.

7 Slavotinek AM: Novel microdeletion syndromes detected by chromosome microarrays. Hum Genet 2008; 124: 1-17.

8 Shaw-Smith C, Pittman AM, Willatt $L$ et al: Microdeletion encompassing MAPT at chromosome $17 q 21.3$ is associated with developmental delay and learning disability. Nat Genet 2006; 38: 1032-1037.

9 Koolen DA, Sharp AJ, Hurst JA et al: Clinical and molecular delineation of the 17q21.31 microdeletion syndrome. J Med Genet 2008; 45: 710-720.

10 Sharp AJ, Hansen S, Selzer RR et al: Discovery of previously unidentified genomic disorders from the duplication architecture of the human genome. Nat Genet 2006; 38: 1038-1042

11 Brunetti-Pierri N, Berg JS, Scaglia F et al: Recurrent reciprocal 1q21.1 deletions and duplications associated with microcephaly or macrocephaly and developmental and behavioral abnormalities. Nat Genet 2008; 40: 1466-1471.

12 Mefford HC, Sharp AJ, Baker C et al: Recurrent rearrangements of chromosome 1q21.1 and variable pediatric phenotypes. N Engl J Med 2008; 359. 1685-1699

13 Ullmann R, Turner G, Kirchhoff M et al: Array CGH identifies reciprocal 16p13.1 duplications and deletions that predispose to autism and/or mental retardation. Hum Mutat 2007; 28: 674-682.

14 Sharp AJ, Mefford HC, Li K et al: A recurrent $15 q 13.3$ microdeletion syndrome associated with mental retardation and seizures. Nat Genet 2008; 40: 322-328.

15 Stefansson H, Rujescu D, Cichon S et al: Large recurrent microdeletions associated with schizophrenia. Nature 2008; 455: 232-236

16 Stone JL, O'Donovan MC, Gurling $\mathrm{H}$ et al: Rare chromosomal deletions and duplications increase risk of schizophrenia. Nature 2008; 455: 237-241.

17 Ben-Shachar S, Lanpher B, German JR et al: Microdeletion 15q13.3: a locus with incomplete penetrance for autism, mental retardation, and psychiatric disorders. J Med Genet 2009; 46: 382-388.

18 Dibbens LM, Mullen S, Helbig I et al: Familial and sporadic 15q13.3 microdeletions in idiopathic generalized epilepsy: precedent for disorders with complex inheritance. Hum Mol Genet 2009; 18: 3625-3631.

19 Helbig I, Mefford HC, Sharp AJ et al: 15q13.3 microdeletions increase risk of idiopathic generalized epilepsy. Nat Genet 2009; 41: 160-162.

20 Hannes FD, Sharp AJ, Mefford HC et al: Recurrent reciprocal deletions and duplications of $16 \mathrm{p} 13.11$ : the deletion is a risk factor for MR/MCA while the duplication may be a rare benign variant. J Med Genet 2009; 46: 223-232.

21 van Bon BW, Mefford HC, Menten B et al: Further delineation of the $15 \mathrm{q} 13$ microdeletion and duplication syndromes: a clinical spectrum varying from non-pathogenic to a severe outcome. J Med Genet 2009; 8: 511-523.
22 Pagnamenta AT, Wing K, Akha ES et al: A 15q13.3 microdeletion segregating with autism. Eur J Hum Genet 2009; 5: 687-692.

23 Van den Veyver I, Patel A, Shaw CA et al: Clinical use of array comparative genomic hybridization (aCGH) for prenatal diagnosis in 300 cases. Prenat Diagn 2009; 29: 29-39.

24 Balciuniene J, Feng N, lyadurai $\mathrm{K}$ et al: Recurrent 10q22-q23 deletions: a genomic disorder on 10q associated with cognitive and behavioral abnormalities. Am J Hum Genet 2007; 80: 938-947.

25 Lupski JR, Stankiewicz P: Genomic disorders: molecular mechanisms for rearrangements and conveyed phenotypes. PLoS Genet 2005; 1: e49.

26 Alliman S, Coppinger J, Marcadier J et al: Clinical and molecular characterization of individuals with recurrent genomic disorder at 10q22.3q23.2. Clin Genet 2010; 78: 162-168.

27 Lupski JR: Genomic disorders ten years on. Genome Med 2009; 1: 42.

28 Stevenson RE, Procopio-Allen AM, Schroer RJ et al: Genetic syndromes among individuals with mental retardation. Am J Med Genet A 2003; 123A: 29-32.

29 Walters RG, Jacquemont S, Valsesia A et al: A new highly penetrant form of obesity due to deletions on chromosome 16p11.2. Nature 2010; 463: 671-675.

30 Lupski JR: Genomic disorders: structural features of the genome can lead to DNA rearrangements and human disease traits. Trends Genet 1998; 14: 417-422.

31 Mefford HC, Eichler EE: Duplication hotspots, rare genomic disorders, and common disease. Curr Opin Genet Dev 2009; 19: 196-204.

32 Shaffer LG, Bejjani BA, Torchia B et al: The identification of microdeletion syndromes and other chromosome abnormalities: cytogenetic methods of the past, new technologies for the future. Am J Med Genet C Semin Med Genet 2007; 145C: 335-345.

33 Bayes M, Magano LF, Rivera $\mathrm{N}$ et al: Mutational mechanisms of Williams-Beuren syndrome deletions. Am J Hum Genet 2003; 73: 131-151.

$34 \mathrm{Gu} \mathrm{W}$, Zhang F, Lupski JR: Mechanisms for human genomic rearrangements. Pathogenetics 2008; 1: 4

35 Shaw CJ, Bi W, Lupski JR: Genetic proof of unequal meiotic crossovers in reciprocal deletion and duplication of 17p11.2. Am J Hum Genet 2002; 71: 1072-1081.

36 Makoff AJ, Flomen RH: Detailed analysis of 15q11-q14 sequence corrects errors and gaps in the public access sequence to fully reveal large segmental duplications at breakpoints for Prader-Willi, Angelman, and inv dup(15) syndromes. Genome Biol 2007; 8: R114.

37 Leggett $\mathrm{V}$, Jacobs $\mathrm{P}$, Nation $\mathrm{K}$ et al: Neurocognitive outcomes of individuals with a sex chromosome trisomy: XXX, XYY, or XXY: a systematic review. Dev Med Child Neurol 2010; 52: 119-129.

38 Delnatte C, Sanlaville D, Mougenot JF et al: Contiguous gene deletion within chromosome arm $10 \mathrm{q}$ is associated with juvenile polyposis of infancy, reflecting cooperation between the BMPR1A and PTEN tumor-suppressor genes. Am J Hum Genet 2006; 78: 1066-1074.

39 Eng C: PTEN: one gene, many syndromes. Hum Mutat 2003; 22: 183-198.

40 Howe JR, Bair JL, Sayed MG et al: Germline mutations of the gene encoding bone morphogenetic protein receptor $1 \mathrm{~A}$ in juvenile polyposis. Nat Genet 2001; 28: 184-187.

41 Shapiro SD, Hansen KL, Pasztor LM et al: Deletions of the long arm of chromosome 10. Am J Med Genet 1985; 20: 181-196.

42 Menko FH, Kneepkens CM, de LN et al: Variable phenotypes associated with 10q23 microdeletions involving the PTEN and BMPR1A genes. Clin Genet 2008; 74: 145-154.

43 Salviati L, Patricelli M, Guariso G et al: Deletion of PTEN and BMPR1A on chromosome 10q23 is not always associated with juvenile polyposis of infancy. Am J Hum Genet 2006; 79. 593-596.

44 Zigman AF, Lavine JE, Jones MC et al: Localization of the Bannayan-Riley-Ruvalcaba syndrome gene to chromosome 10q23. Gastroenterology 1997; 113: 1433-1437.

45 Sweet K, Willis J, Zhou XP et al: Molecular classification of patients with unexplained hamartomatous and hyperplastic polyposis. JAMA 2005; 294: 2465-2473.

46 Gaussin V, Van de PT, Mishina Y et al: Endocardial cushion and myocardial defects after cardiac myocyte-specific conditional deletion of the bone morphogenetic protein receptor ALK3. Proc Natl Acad Sci USA 2002; 99: 2878-2883.

47 Yamazaki M, Araki K, Shibata A et al: Molecular cloning of a cDNA encoding a novel member of the mouse glutamate receptor channel family. Biochem Biophys Res Commun 1992; 183: 886-892.

48 Vasan RS, Glazer NL, Felix JF et al: Genetic variants associated with cardiac structure and function: a meta-analysis and replication of genome-wide association data. JAMA 2009; 302: 168-178.

49 Treutlein J, Muhleisen TW, Frank J et al: Dissection of phenotype reveals possible association between schizophrenia and glutamate receptor delta 1 (GRID1) gene promoter. Schizophr Res 2009; 111: 123-130.

50 Guo SZ, Huang K, Shi YY et al: A case-control association study between the GRID1 gene and schizophrenia in the Chinese Northern Han population. Schizophr Res 2007; 93: 385-390.

51 Zhu Y, Kalbfleisch T, Brennan MD et al: A microRNA gene is hosted in an intron of a schizophrenia-susceptibility gene. Schizophr Res 2009; 109: 86-89.

52 Fallin MD, Lasseter VK, Avramopoulos D et al: Bipolar I disorder and schizophrenia: a 440-single-nucleotide polymorphism screen of 64 candidate genes among Ashkenazi Jewish case-parent trios. Am J Hum Genet 2005; 77: 918-936.

53 Monaghan DT, Bridges RJ, Cotman CW: The excitatory amino acid receptors: their classes, pharmacology, and distinct properties in the function of the central nervous system. Annu Rev Pharmacol Toxicol 1989; 29: 365-402. 
54 Kim JS, Kornhuber HH, Schmid-Burgk W et al: Low cerebrospinal fluid glutamate in schizophrenic patients and a new hypothesis on schizophrenia. Neurosci Lett 1980; 20: 379-382.

55 Coyle JT: Glutamate and schizophrenia: beyond the dopamine hypothesis. Cell Mol Neurobiol 2006; 26: 365-384.

56 Chen PL, Avramopoulos D, Lasseter VK et al: Fine mapping on chromosome 10q22q23 implicates neuregulin 3 in schizophrenia. Am J Hum Genet 2009; 84: 21-34.

57 Fallin MD, Lasseter VK, Wolyniec PS et al: Genomewide linkage scan for schizophrenia susceptibility loci among Ashkenazi Jewish families shows evidence of linkage on chromosome 10q22. Am J Hum Genet 2003; 73: 601-611.

58 Faraone SV, Hwu HG, Liu CM et al: Genome scan of Han Chinese schizophrenia families from Taiwan: confirmation of linkage to 10q22.3. Am J Psychiatry 2006; 163: 1760-1766.

59 Liu J, Juo SH, Dewan A et al: Evidence for a putative bipolar disorder locus on 2p13-16 and other potential loci on 4q31, 7q34, 8q13,9q31,10q21-24, 13q32, 14q21 and 17q11-12. Mol Psychiatry 2003; 8: 333-342.

60 Falls DL: Neuregulins: functions, forms, and signaling strategies. Exp Cell Res 2003; 284: 14-30.

61 Shaikh TH, Gai X, Perin JC et al: High-resolution mapping and analysis of copy number variations in the human genome: a data resource for clinical and research applications. Genome Res 2009; 19: 1682-1690.

62 Howard B, Panchal H, McCarthy A et al: Identification of the scaramanga gene implicates neuregulin3 in mammary gland specification. Genes Dev 2005; 19 : 2078-2090.

63 Howard BA: The role of NRG3 in mammary development. J Mammary Gland Biol Neoplasia 2008; 13: 195-203.

64 Erdogan F, Belloso JM, Gabau E et al: Fine mapping of a de novo interstitial 10q22 q23 duplication in a patient with congenital heart disease and microcephaly. Eur J Med Genet 2008; 51: 81-86.
65 Dufke A, Singer S, Borell-Kost S et al: De novo structural chromosomal imbalances: molecular cytogenetic characterization of partial trisomies. Cytogenet Genome Res 2006; 114: 342-350.

66 Han JY, Kim KH, Jun HJ et al: Partial trisomy of chromosome 10(q22-q24) due to maternal insertional translocation (15;10). Am J Med Genet A 2004; 131: 190-193.

67 Goss PW, Voullaire L, Gardner RJ: Duplication 10q22.1-q25.1 due to intrachromosomal insertion: a second case. Ann Genet 1998; 41: 161-163.

68 Donald-McGinn DM, Kirschner R, Goldmuntz E et al: The Philadelphia story: the 22q11.2 deletion: report on 250 patients. Genet Couns 1999; 10: 11-24.

69 Digilio MC, Marino B, Giannotti A et al: Familial deletions of chromosome 22q11. Am J Med Genet 1997; 73: 95-96.

70 Ben-Shachar S, Ou Z, Shaw CA et al: 22q11.2 distal deletion: a recurrent genomic disorder distinct from DiGeorge syndrome and velocardiofacial syndrome. Am J Hum Genet 2008; 82: 214-221.

71 Rodningen OK, Prescott T, Eriksson AS et al: 1.4Mb recurrent 22q11.2 distal deletion syndrome, two new cases expand the phenotype. Eur J Med Genet 2008; 51: 646-650.

72 Berg JS, Brunetti-Pierri N, Peters SU et al: Speech delay and autism spectrum behaviors are frequently associated with duplication of the $7 q 11.23$ Williams-Beuren syndrome region. Genet Med 2007; 9: 427-441.

73 Shinawi M, Liu P, Kang SH et al: Recurrent reciprocal 16p11.2 rearrangements associated with global developmental delay, behavioral problems, dysmorphism, epilepsy, and abnormal head size. J Med Genet 2009; 47: 332-341.

74 Christian SL, Robinson WP, Huang B et al: Molecular characterization of two proximal deletion breakpoint regions in both Prader-Willi and Angelman syndrome patients. Am J Hum Genet 1995; 57: 40-48.

75 Ballif BC, Hornor SA, Jenkins $\mathrm{E}$ et al: Discovery of a previously unrecognized microdeletion syndrome of 16p11.2-p12.2. Nat Genet 2007; 39: 1071-1073.

Supplementary Information accompanies the paper on European Journal of Human Genetics website (http://www.nature.com/ejhg) 